\title{
Dual-Stage Piezoelectric Nano-Positioner Utilizing a Range-Extended Optical Fiber Fabry-Perot Interferometer
}

\author{
Shao-Kang Hung, Student Member, IEEE, En-Te Hwu, Mei-Yung Chen, and Li-Chen Fu, Fellow, IEEE
}

\begin{abstract}
This paper proposes a new modulation scheme using high-order harmonic information to solve the so-called ambiguity problem of interferometry. To start with, we build a fiber FabryPerot interferometer to serve as a displacement sensor, which has two operation modes-coarse and fine modes. Integrating the afore-developed sensor, a piezoelectric actuator, and a scheduled proportional-integral/adaptive-sliding controller, we construct a dual-stage nanopositioning system. The experimental results show that the proposed system has the capability to extend the positioning range beyond the limit of the wavelength while keeping the naturally high resolution, $\pm \mathbf{5} \mathbf{~ m}$, of interferometry.
\end{abstract}

Index Terms-Adaptive control, interferometry, modulation, nanopositioning, optical fiber.

\section{INTRODUCTION}

I NTERFEROMETRY has excellent sensitivity [1], and is used in various measurements such as temperature [2], strain [3], magnetic field [4], angular velocity [5], and many other physical parameters [6]. The largest advantage of optical interferometer is also its main disadvantage. Due to the short wavelength of light, the sensitivity of a single-wavelength interferometer is very high, but the dynamic range is limited unless additional information is available. The phase of the light, and hence, the phase of the interference pattern, repeats itself at distance intervals equal to the wavelength in a singlewavelength interferometric measurement. Thus, an optical path difference (OPD) gives identical interferometric measurement as ( $m \lambda+\mathrm{OPD}$ ), where $m$ is a positive integer and $\lambda$ is the wavelength. It is the so-called ambiguity problem of interferometer. In order to extend the measurement range, four classifications of methods were proposed: 1) white-light interferometry [7]; 2) dual-wavelength inteferometry [8], [9]; 3) phase-shifting interferometry [3]; and 4) modulation techniques [10], [11]. If a white light source is used in an interferometer, the best contrast interference fringes are obtained only when the two paths in the interferometer are equal. Dual-wavelength method is to perform the measurement at two wavelengths, and compare the

Manuscript received March 1, 2006; revised December 15, 2006. Recommended by Guest Editors H.-P. Huang and F.-T. Cheng. This work was supported in part by the National Science Council, R.O.C., under Grant NSC-94-2752-E002-007-PAE.

S.-K. Hung and L.-C. Fu are with the Department of Electrical Engineering, National Taiwan University, Taipei 10617, Taiwan, R.O.C. (e-mail: skhung@ntu.edu.tw; lichen@ntu.edu.tw).

E.-T. Hwu is with the Department of Mechanical Engineering, National Taiwan University, Taipei 10617, Taiwan, R.O.C. (e-mail: whoand@gmail.com).

M.-Y. Chen is with the Department of Industrial Education, National Taiwan Normal University, Taipei 10617, Taiwan, R.O.C. (e-mail: cmy@ntnu.edu.tw).

Digital Object Identifier 10.1109/TMECH.2007.897264 measurement data for the different wavelengths to determine the true OPD. The optical phase is a function of the wavelength and OPD. Thus, modulation can be implemented on these two variables by oscillating them at a relatively high frequency. It is difficult to change the wavelength of a laser device without changing its intensity [11]. More complicated optical and electrical equipments are needed to compensate the unstable intensity and wavelength fluctuation of the laser source.

This paper chooses the displacement modulation method [12] to break the ambiguity. Utilizing high-order harmonic information, solidly digital signals with $90^{\circ}$ phase difference can be obtained to achieve far-reaching measurement. The idea is learned from "incremental encoders [12], [13]," which are widely used for measuring angular position. Combining with the naturally fine resolution of interferometers, a powerful displacement sensor having both long measurement range and high resolution was developed. In Section II, the design of the built fiber Fabry-Perot interferometer (FFPI) is analyzed. In Section III, combining an FFPI with a piezoelectric actuator and the associated controller, a nanopositioner possessing high resolution in displacement, high stiffness, and fast frequency response is proposed. It can be used in precision positioning applications, such as scanning probe microscopy and diamond turning machines [14]-[16]. In Section IV, experimental results are presented. A discussion of the obtained results is provided in Section V. The concluding remarks are given in Section VI.

\section{FFPI DisPlacement SENSOR SySTEM}

\section{A. Working Principle of FFPI}

An FFPI makes use of multiple reflections between two closely spaced partially reflective interfaces. Part of the light is transmitted each time the light reaches the second surface, resulting in multiple beams, which can interfere with each other. The large number of interfering rays produces an interferometer with extremely high resolution, somewhat like the multiple slits of a diffraction grating that increases its resolution.

If the polished optical fiber end is used as the interface of the Fabry-Perot cavity, this kind of device is called an FFPI. Fig. 1 illustrates the configuration of FFPI. The laser diode emits the light into the first arm of a Y-shaped fiber coupler. After the optical interference occurrs at the fiber end of the second arm, the interfered fringe signal can be received by a photodetector installed at the end of third arm.

As the normally incident light reaches the fiber end of second arm, part of it is reflected back by the interface between fiber 


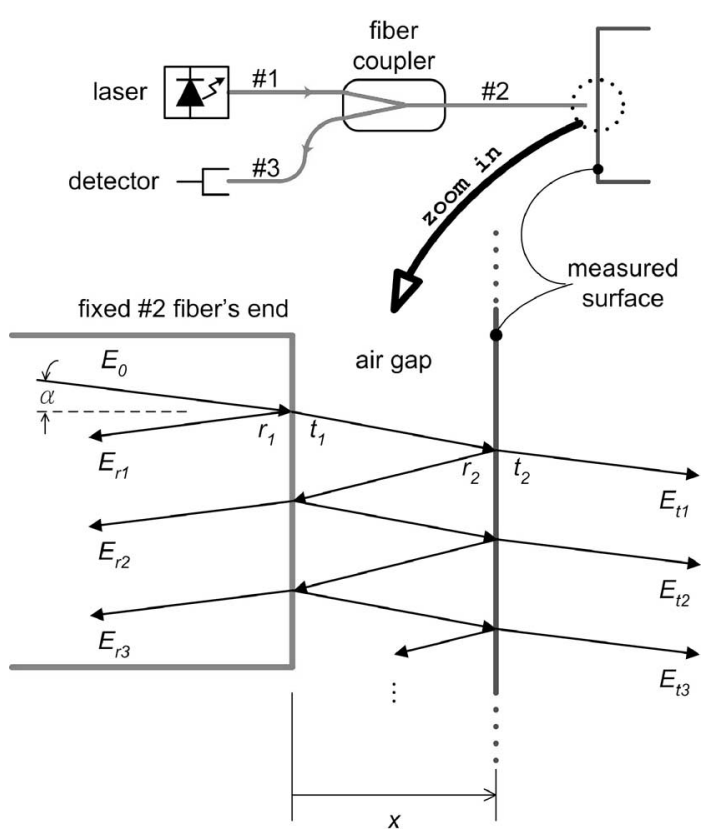

Fig. 1. Configuration of the fiber FFPIs.

and the air while the other part is transmitted. Zero absorption is assumed. The partial reflection/transmission behavior will happen for infinite times. Optical interference can occur in both groups of light. Starting with the intensity of the incident light $I_{0}$, its corresponding electric field is denoted as $E_{0} e^{\omega t}$. The $k$ th reflected electric field from the first interface is denoted as $E_{\mathrm{rk}}$, while the $k$ th partially transmitted electric field through the second interface is denoted as $E_{\mathrm{tk}}$. The coefficients of amplitude reflection $r_{1}$ and transmission $t_{1}$ denote light traveling through the first interface while the coefficients $r_{2}$ and $t_{2}$ denote light traveling through second interface. The multiple output beams differ in phase due to the different path lengths traversed by each of the beams. The optical phase acquired by the light on one round trip through the cavity is given by

$$
\phi=\frac{4 \pi n x \cos \alpha}{\lambda}
$$

where $\lambda$ is the wavelength of the laser; $\alpha$ is the incident angle; $n$ is the refraction index of the cavity; and $x$ is the gap distance between two interfaces.

Since $n=1$ for air and has perpendicular incidence, (1) can be simplified into

$$
\phi=\frac{4 \pi x}{\lambda}
$$

The electric field of each of the transmitted waves can, thus, be written as

$$
\begin{aligned}
E_{t 1} & =\left(E_{0} e^{\varpi t}\right)\left(t_{1} i\right)\left(t_{2} i\right) \\
E_{t 2} & =\left(E_{0} e^{\varpi t}\right)\left(t_{1} i\right)\left(-r_{2}\right)\left(-r_{1}\right)\left(t_{2} i\right) e^{i \phi} \\
E_{t 3} & =\left(E_{0} e^{\varpi t}\right)\left(t_{1} i\right)\left(-r_{2}\right)\left(-r_{1}\right)\left(-r_{2}\right)\left(-r_{1}\right)\left(t_{2} i\right) e^{i 2 \phi} \\
\vdots & \\
E_{t k} & =\left(E_{0} e^{\varpi t}\right) t_{1} t_{2}\left(r_{1} r_{2} e^{i \phi}\right)^{k-1}, \quad k \in N .
\end{aligned}
$$

The electric field of the interfered waves $E_{t}$ is

$$
E_{t}=\sum_{k=1}^{\infty} E_{\mathrm{tk}}=\left(E_{0} e^{\varpi t}\right) \frac{-t_{1} t_{2}}{1-r_{1} r_{2} e^{i \phi}} .
$$

If the two interfaces are the same, we have $t_{1}=t_{2} \equiv t$ and $r_{1}=r_{2} \equiv r$. Introducing the power reflectivity $R=r^{2}$ with lossless assumption $r^{2}+t^{2}=1$, we can find the normalized power transmission as

$$
\frac{I_{t}}{I_{0}}=\left|\frac{E_{t}}{E_{0}}\right|^{2}=\frac{(1-R)^{2}}{1+R^{2}-2 R \cos \phi}
$$

which is known as the "airy function" [17]. On the other hand, the electric field of the reflected group can be expressed as

$$
\begin{aligned}
& E_{r 1}=\left(E_{0} e^{\varpi t}\right)\left(-r_{1}\right) \\
& E_{r 2}=\left(E_{0} e^{\varpi t}\right)\left(t_{1} i\right)\left(-r_{2}\right)\left(t_{1} i\right) e^{i \phi} \\
& E_{r 3}=\left(E_{0} e^{\varpi t}\right)\left(t_{1} i\right)\left(-r_{2}\right)\left(-r_{1}\right)\left(-r_{2}\right)\left(t_{1} i\right) e^{i 2 \phi}
\end{aligned}
$$

$$
E_{\mathrm{rk}}=\left(E_{0} e^{\varpi t}\right) \frac{t_{1}^{2}}{r_{1}}\left(r_{1} r_{2} e^{i \phi}\right)^{k-1}, \quad k \in N-\{1\} .
$$

If the two interfaces are the same, i.e., $r_{1}=r_{2}$, all of $E_{\mathrm{rk}}$ form a geometric sequence. The normalized power reflection can easily be found as [18]

$$
\frac{I_{t}}{I_{0}}=\left|\frac{\sum_{k=1}^{\infty} E_{\mathrm{rk}}}{E_{0}}\right|^{2}=\frac{2 R(1-\cos \phi)}{1+R^{2}-2 R \cos \phi} .
$$

However, if the material of the measured surface is different from the sensing fiber, the geometric relation vanishes. The normalized power reflection should be derived into a more complicated form

$$
\frac{I_{t}}{I_{0}}=\left|\frac{\sum_{k=1}^{\infty} E_{\mathrm{rk}}}{E_{0}}\right|^{2}=\frac{R_{1}+R_{2}-2 \sqrt{R_{1} R_{2}} \cos \phi}{1+R_{1} R_{2}-2 \sqrt{R_{1} R_{2}} \cos \phi}
$$

where $R_{1}$ and $R_{2}$ are the power reflectivity of the first and the second interfaces, respectively. $R_{1}$ is $3.5 \%$ for the interface between glass and air. When the gap distance $x=0, R_{2}$ is 0.9 for the interface between air and the measured object, a golden coated mirror. As the gap distance increases, the power-coupled back to the fiber decreases [19]. The equivalent reflectivity is

$$
R_{2}=0.9\left\{\frac{a}{a+2 x \tan \left[\sin ^{-1}(\mathrm{NA})\right]}\right\}^{2}
$$

where $a$ and NA are the fiber core radius and the numerical aperture, respectively. The transduced signal can be represented by (8). Substituting the parameters of $a=4.15 \mu \mathrm{m}, \mathrm{NA}=0.13$, and $\lambda=1.31 \mu \mathrm{m}$, we have the simulation result in Fig. 2. Further modulator design is based on this mathematical model.

\section{B. Modulation and Demodulation}

In Fig. 2 , the signal oscillates by the period of $\lambda / 2$. Local maximum represents constructive interference while local minimum means destructive interference. As the gap distance $x$ increases, the amplitude decreases. In fact, the dc value also 




Fig. 2. Plot of $I_{r} / I_{0}$ versus gap distance $x$.

slightly changes with $x$. The received signal can be approximated as

$$
I_{r}=A(x) \cdot\left[-\cos \left(\frac{4 \pi x}{\lambda}\right)\right]+D(x)
$$

where $A(x)$ and $D(x)$ represent the variant amplitude and dc value, respectively. Thus, it is difficult to reveal $x$ from the ambiguous fringe signal directly. In order to get rid of this predicament, we design two terms

$$
\left\{\begin{array}{c}
g \equiv \operatorname{sign}\left(\frac{d I_{r}}{d x}\right) \\
h \equiv \operatorname{sign}\left(\frac{d^{2} I_{r}}{d x^{2}}\right) .
\end{array}\right.
$$

In order to obtain $g$ and $h$ signals, the phase-sensitive detection technique is adopted [20]. A relatively high-frequency sinusoidal signal is applied on a small piezoelectric stack to oscillate the fiber end slightly. This modulating action will induce certain response on the signal $I_{r}$ at the identical frequency. The lock-in amplifier (SR830, Stanford Research Systems, Inc.) can resolve such little response, and give an output phase signal. If the response and the modulation signal are in-phase or out-of-phase, the output phase signal is positive or negative, respectively. Obviously, this output phase signal is the previously defined $g$-signal. The lock-in amplifier can also give the secondharmonic phase signal. That is, the source of the $h$-signal can be obtained.

The following simulation demonstrates the functionality of these two terms in (11). In Fig. 3, as $x$ increases, the $g$-signal lags the $h$-signal by $90^{\circ}$. On the contrary, the $g$-signal leads the $h$-signal by $90^{\circ}$ when $x$ moves to the opposite direction. This coding characteristic is the same with "incremental encoders," which are widely used to measure angular or linear position. While the $g$ - and $h$-signals form a quadrant system, we can notice that the quadrant length is $\lambda / 8$ instead of $\lambda / 4$ because OPD doubles $x$ due to light's round-trip reflection. Both $g$ and $h$-signals are fed into an accurate direction-detecting circuit [13]. Counting the output up/down clocks, we can reveal $x$ with the resolution of $\lambda / 8,163.75 \mathrm{~nm}$. It is labeled as coarse mode. Since the direction-detecting circuit and the counter only consist of logic components, the response speed can be fast. The naturally fine resolution of the interferometer is not used so far.
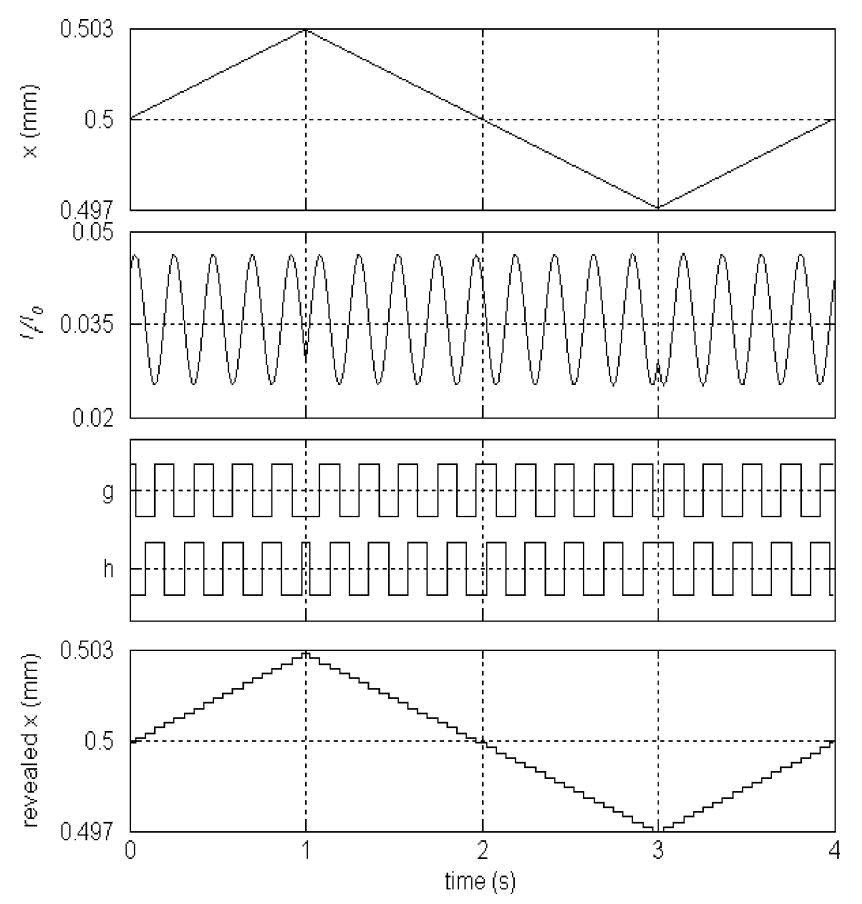

Fig. 3. Functionality of $g$-signal and $h$-signal. As $x$ changes its direction, the lead-lag relation between $g$-signal and $h$-signal changes correspondingly.

Next, we turn to deal with the original signal of $I_{r}$. Using coarse mode to prescan a wide range of $x$, we can find enough accurate $A(x)$ and $D(x)$, since they do not have great gradient values. A new signal can be defined as

$$
f \equiv\left[-\cos \left(\frac{4 \pi x}{\lambda}\right)\right]=\frac{\left[I_{r}-D(x)\right]}{A(x)} \in[-1,1] .
$$

Then, we can reveal

$$
x=\left\{\begin{array}{cc}
\frac{\lambda}{4 \pi} \cos ^{-1}(-f), & \text { if } h>0 \\
\pi+\frac{\lambda}{4 \pi} \cos ^{-1}(f), & \text { if } h<0 .
\end{array}\right.
$$

Bundled $(g, h)$ signal also gives the quadrant information of $f$-signal. It can be observed in Fig. 3 that $(+,+)$ indicates the first quadrant, $(-,+)$ indicates the second quadrant, etc. By knowing the exact quadrant information, high resolution $x$ can be revealed from the $f$-signal utilizing inverse-trigonometry [3] in (13). In order to pursue extreme resolution, a low-pass filter is adopted to suppress the noise on $f$-signal. Further experiment shows that the resolution of such "fine mode" can reach $\pm 5 \mathrm{~nm}$. Hence, a displacement sensor having both long measurement range and high resolution is developed by fusion of coarse mode and fine mode.

\section{Controller Design}

We design two individual controllers for coarse mode and fine mode, since their characteristics are much different. Fine mode has higher resolution but slower response than coarse mode. A classical proportional-integral (PI) controller is chosen for coarse mode while an adaptive sliding-mode controller is 
designed for fine mode. Rate limiters are attached at the outputs of the controllers in order not to drive the actuator beyond the maximum sensing speed of the FFPI. A superior scheduler is also designed to switch the operation between coarse mode and fine mode.

\section{A. Adaptive Sliding-Mode Controller}

In order to design a controller that possesses a good capability to deal with piezoelectric nonlinearity, uncertainty, high robustness, and self-tuning property, we propose an adaptive sliding-mode controller for the FFPI's fine-mode operation. To start with, a linear second-order model can be used to represent the actuator's nominal dynamics as

$$
\ddot{x}+a_{1} \dot{x}+a_{0} x=b u
$$

where $x$ is the displacement of the actuator, $b$ is the forcing coefficient of the control input $u, a_{1}$ and $a_{0}$ are the damping and the stiffness of the system, respectively. The parameters $a_{1}, a_{0}$, and $b$ can be well estimated via an offline identification test.

Then, the system dynamics can be transformed into error coordinates using the relation $e=x-x_{d}$, where $x_{d}$ represents the desired trajectory. Using the compact notations $A=\left[-a_{1}-\right.$ $\left.a_{0}\right] ; X=[\dot{x} x]^{T}$, we have

$$
\ddot{e}=A X+b u-x_{d} .
$$

Nevertheless, we add an additional term to represent the noise, the disturbance, and the actuator's piezoelectric hysteresis behavior as

$$
\ddot{e}=A X+b u-x_{d}+w
$$

where $w$ is assumed to be bounded. Then, we divide this term into two parts, one is a constant uncertainty $w_{c}$ and the other one is a varying uncertainty $w_{v}$, which is assumed bounded and satisfies $\left|w_{v}\right| \leq w_{\max }$. Therefore, (16) can be expressed as

$$
\ddot{e}=A X+b u-x_{d}+w_{c}+w_{v} .
$$

A first-order sliding surface $s$ is chosen such that

$$
s=\dot{e}+\gamma e
$$

where $\gamma$ is a positive scalar to be designed. The sliding surface is designed such that the system is stable when the system state lies on this surface. Therefore, the problem is reduced to guarantee that the state reaches the sliding surface, or in the case of bounded tracking, some region on the surface. Our main purpose is to force the tracking error to zero. Given the initial condition $x_{d}(0)=x(0)$, with the initial desired state $x_{d}(0)$, the tracking problem $x(t) \rightarrow x_{d}(t)$ as $t \rightarrow \infty$, is equivalent to that of those remaining on the surface $s$ for all $t>0$; indeed, $s \equiv 0$ represents a linear differential equation whose unique solution is $e \equiv 0$. Then, the sliding surface dynamics can be determined by taking the time derivative of (18), i.e.,

$$
\dot{s}=\ddot{e}+\gamma \dot{e} .
$$

Then, substituting (17) and (18) into (19), we have

$$
\dot{s}=A X+b u-\ddot{x}_{d}+\gamma \dot{e}+w_{c}+w_{v} .
$$

Based on (20), the control law is designed as

$$
u_{\mathrm{AS}}=\hat{b}^{-1}\left(-A X+\ddot{x}_{d}-\gamma \dot{e}-\hat{w}_{c}-\kappa s-\eta \operatorname{sat}(s)\right)
$$

where $\kappa>0$ and $\eta>\left|w_{\max }\right|$ are maneuverable control parameters. Moreover, $\hat{b}$ and $\hat{w}_{c}$ are the estimated values of $b$ and $w_{c}$, respectively, and sat(.) is the saturation function defined as

$$
\operatorname{sat}(s) \equiv \begin{cases}1, & \text { if } \varepsilon<s \\ \frac{s}{|\varepsilon|}, & \text { if }-\varepsilon \leq s \leq \varepsilon \\ -1, & \text { if } s<-\varepsilon\end{cases}
$$

where $\varepsilon$ is the width of boundary layer.

Substituting (21) into (16), we can obtain

$$
\begin{aligned}
\ddot{e}= & A X+b\left\{\hat{b}^{-1}\left[-A X+x_{d}-\gamma \dot{e}-\hat{w}_{c}-\kappa s-\eta \operatorname{sat}(s)\right]\right\} \\
& -\ddot{x}_{d}+w_{c}+w_{v} \\
= & A X+(\tilde{b}+\hat{b})\left\{\hat { b } ^ { - 1 } \left[-A X+x_{d}-\gamma \dot{e}-\hat{w}_{c}-\kappa s\right.\right. \\
& -\eta \operatorname{sat}(s)]\}-\ddot{x}_{d}+w_{c}+w_{v} \\
= & \tilde{b} u_{\mathrm{AS}}-\gamma \dot{e}-\kappa s-\eta \operatorname{sat}(s)+\tilde{w}_{c}+w_{v}
\end{aligned}
$$

where the estimation errors are defined as $\tilde{b}=b-\hat{b}$, and $\tilde{w}_{c}=$ $w_{c}-\hat{w}_{c}$. By applying appropriate gains $\kappa, \eta$, and $\gamma$, we can accelerate the convergence, and force the error to zero in a shorter period of time.

Defining a Lyapunov function candidate $V$, which is a positive definite function, we have

$$
V=\frac{1}{2} s^{2}+\frac{1}{2} \tilde{b}^{2} \tau_{1}^{-1}+\frac{1}{2} \tilde{w}^{2} \tau_{2}^{-1}
$$

where $\tau_{1}$ and $\tau_{2}$ are positive constants. The time derivative of $V$ can be found as

$$
\dot{V}=s \dot{s}+\tilde{\tilde{b}} \tau_{1}^{-1}+\tilde{w} \dot{\tilde{w}} \tau_{2}^{-1} .
$$

As described in (18)-(22), (25) can be expressed as

$$
\begin{aligned}
\dot{V}= & -s^{2} \kappa-s\left(\eta \operatorname{sat}(s)-w_{v}\right) \\
& +s \tilde{b} u_{\mathrm{AS}}+s \tilde{w}_{c}+\tilde{b} \tau_{1}^{-1} \dot{\tilde{b}}+\tilde{w}_{c} \tau_{2}^{-1} \dot{\tilde{w}}_{c} \\
= & -s^{2} \kappa-s\left(\eta \operatorname{sat}(s)-w_{v}\right) \\
& +\left[\tilde{b}\left(\tau_{1}^{-1} \dot{\tilde{b}}+s u_{\mathrm{AS}}\right)\right]+\left[\tilde{w}_{c}\left(\tau_{2}^{-1} \dot{\tilde{w}}_{c}+s\right)\right] .
\end{aligned}
$$

Using $\sigma$-modification [21] to establish boundedness in the presence of modeling error term, the adaptation laws are derived as

$$
\left\{\begin{array}{c}
\dot{\hat{b}}=-\dot{\tilde{b}}=\tau_{1} s u_{\mathrm{AS}}+\tau_{1} \sigma_{1} \hat{b} \\
\dot{\hat{w}}_{c}=-\dot{\tilde{w}}_{c}=\tau_{2} s+\tau_{2} \sigma_{2} \hat{w}_{c}
\end{array}\right.
$$

where $\sigma_{1}$ and $\sigma_{2}$ are positive adaptive gains. In summary, the proposed adaptive sliding-mode controller is formulated, and its stability can be further proven [22]. 


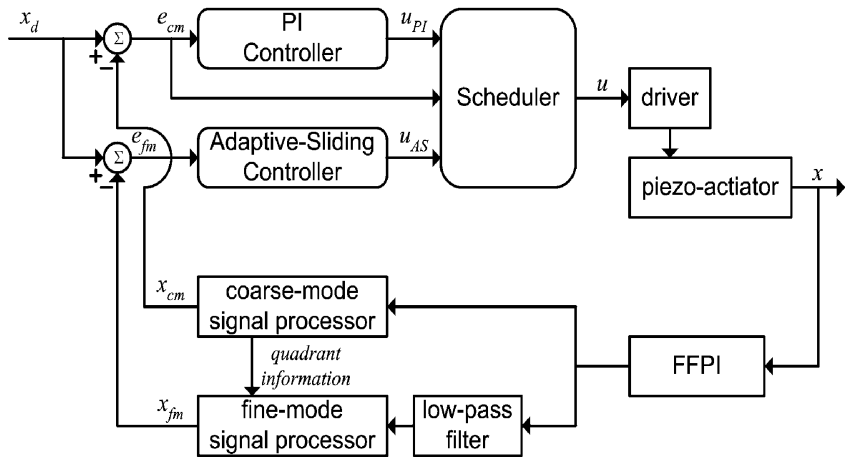

Fig. 4. Architecture of the dual-stage controller.

\section{B. Scheduling Controller}

As afore mentioned, a low-pass filter is used in fine mode to achieve high resolution, but it also slow downs the measurement speed of fine mode. If we face a high resolution $( \pm 5 \mathrm{~nm})$ tracking task, there is no reason to use coarse mode. However, for a regulation task, which requires its transient response to be fast but not necessary to be accurate, the use of coarse mode is thus, motivated. In this section, we design a superior scheduler to deal with regulation tasks. If the error is greater than $163.75 \mathrm{~nm}$, the output of FFPI's coarse mode $x_{\mathrm{cm}}$ is fed to the associated PI controller, and the scheduler assigns $u$ to $u_{\mathrm{PI}}$. In the other case, if the error is less than or equal to $163.75 \mathrm{~nm}$ (i.e., the resolution of the coarse mode), we should switch to the fine mode to achieve better resolution. The behavior of the scheduler is expressed in (28) and Fig. 4.

$$
u= \begin{cases}u_{\mathrm{PI}}, & \text { if } e_{\mathrm{cm}}>163.75 \mathrm{~nm} \\ u_{\mathrm{AS}}, & \text { if } e_{\mathrm{cm}} \leq 163.75 \mathrm{~nm}\end{cases}
$$

where

$$
u_{\mathrm{PI}}=K_{P} e+K_{I} \int e d t
$$

and $K_{P}$ and $K_{I}$ are the P-gain and I-gain of the PI controller, respectively.

\section{EXPERIMENTS}

\section{A. FFPI Test}

The experimental setup is illustrated in Fig. 5. The measurand is a mirror with both coated sides. One side is detected by a commercial displacement sensor (laser-based triangulation measurement system MICROTRAKTM 7000, MTI Instruments, Inc.) while the other side is detected by our FFPI system. As the piezoelectric actuator carries the mirror to move left and right, we can compare the results from these two sensors.

The function generator drives the piezoelectric oscillator to have a peak-to-peak 10 -nm sinusoidal motion at $10 \mathrm{kHz}$. The optical fiber end as the sensing head of our FFPI system is attached on the piezoelectric oscillator for the purpose of displacement modulation. The laser diode emits 1310-nm-wavelength light into the optical fiber coupler. The photodetector transforms the optical signal into the electrical signal, which represents the interference. The two inputs of the lock-in amplifier \#1 are the

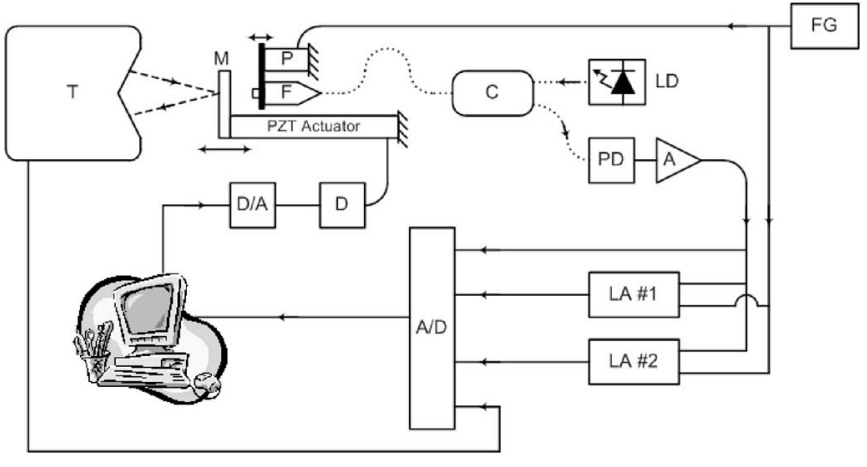

Fig. 5. Block diagram of the proposed FFPI system. Solid lines represent electrical routing. Dashed lines represent the laser path of T. Dotted lines represent the optical fibers. T: triangular displacement sensor; FG: function generator; M: mirror with two coated sides; A: amplifier; P: piezoelectric oscillator; LA: lock-in amplifier; F: fiber connector; D: piezo driver; C: fiber coupler. A/D: A to $\mathrm{D}$ converter; $\mathrm{LD}$ : laser diode; $\mathrm{D} / \mathrm{A}$ : D-to-A converter. PD: photodetector.

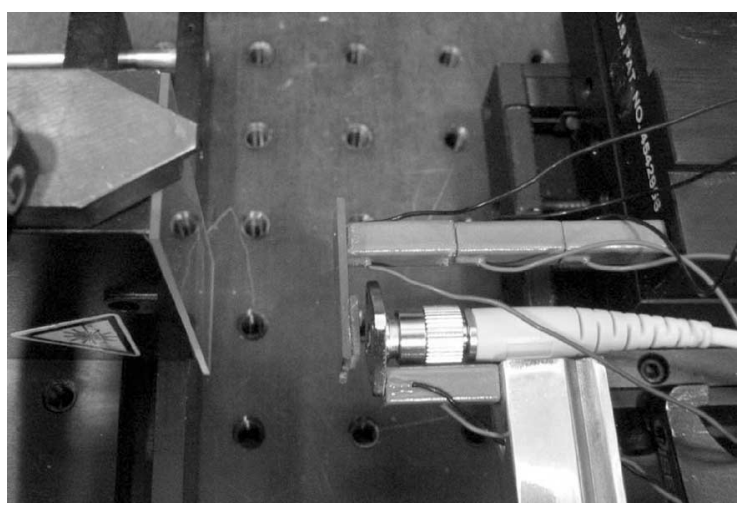

Fig. 6. Photograph of the experimental equipments.

interference signal $I_{r}$ and the reference signal from the signal generator. The output of the lock-in amplifier \#1 is the relative phase difference between the two inputs. The lock-in amplifier \#2 provides the second-harmonic phase information. After appropriate amplifying and conditioning, the data acquisition card (PCI-DAS1602/16, Measurement Computing, Inc.) collects four signals into the computer system. The photograph of the proposed system is shown in Fig. 6.

\section{B. Positioner Test}

After testing the functionality of the sensor, the performance of the whole positioner system is tested in this section. In order to achieve the overall fast positioning performance, we use dual-stage controller. The scheduler switches to coarse mode combined with a fast controller to utilize its fast response, when the target is far away (i.e., the absolute value of the tracking error greater than $163.75 \mathrm{~nm}$ ). If the target is closed, the scheduler switches to fine mode combined with a slow controller to eliminate the residue error slowly and accurately. Referring to the experimental results in Fig. 7, a series of regulation tasks are carried out with the stepping size of $5 \mu \mathrm{m}$. The enlarged figure is also shown to see the details. From 50 to $56 \mathrm{~ms}$, coarse mode and its associated PI controller are selected by the scheduler because the error is still greater than the resolution of coarse 




Fig. 7. Step response of the proposed positioning system. From 50 to $56 \mathrm{~ms}$, coarse mode and its associated PI controller are activated. From 56 to $100 \mathrm{~ms}$, the scheduler activates the adaptive sliding controller. The steady-state error is within $\pm 5 \mathrm{~nm}$.

mode, i.e., $163.75 \mathrm{~nm}$. Therefore, the readout in this stage is quantized by this value. From 56 to $100 \mathrm{~ms}$, the scheduler activates the adaptive sliding controller, which uses the information from fine-mode processor. The readout in this stage is much finer than coarse mode. After a little chattering during 56 to $60 \mathrm{~ms}$, the steady-state resolution can be observed as $\pm 5 \mathrm{~nm}$. With regard to other time domain characteristics in this case, the overshoot is $0.5 \%$, the rise time is $6 \mathrm{~ms}$, and the $\pm 0.1 \%$ settling time is $10 \mathrm{~ms}$. According to the extensive measurement data, the repeatability of the system is in the range of $\pm 5 \mathrm{~nm}$ under the condition of steady-controlled $25^{\circ} \mathrm{C}$ environment temperature.

\section{DISCUSSION}

There might be three forms of interferometer misalignment as shown in Fig. 8. In case 1, the normal vector of the effective test surface $\vec{M}$ is parallel to the displacement vector $\vec{D}$, but not parallel to the propagation vector of light $\vec{L}$. The interferometer-
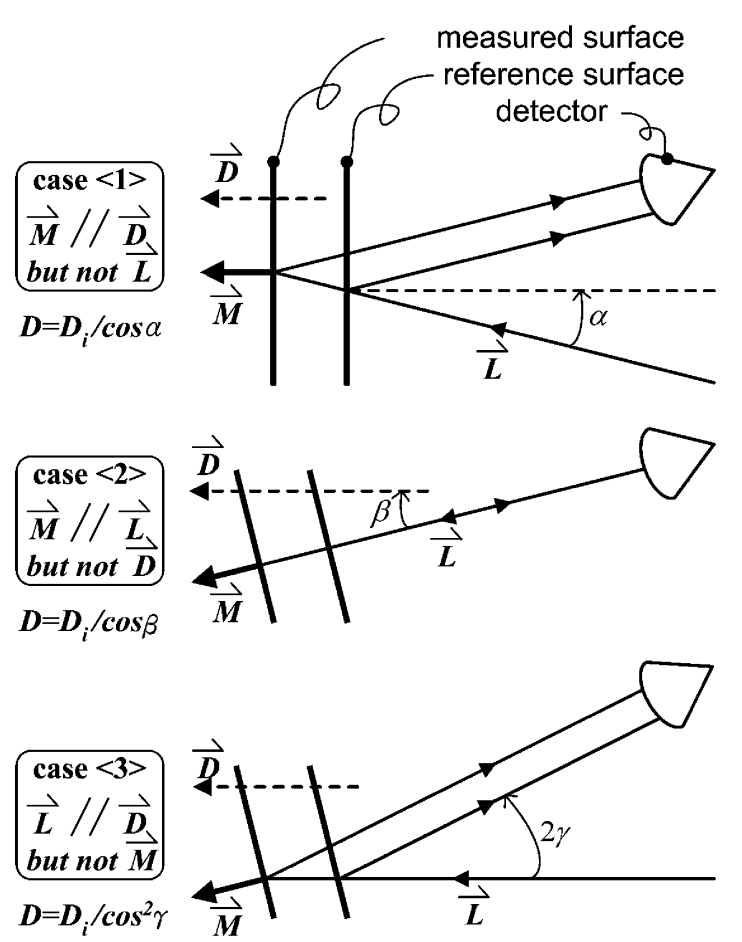

Fig. 8. Three cases of misalignment of interferometry. $\vec{L}$ is the propagation vector of light; $\vec{M}$ is the normal vector of the measured surface; and $\vec{D}$ is the displacement vector of the measured surface. $D_{i}$ is interferometer-measured displacement and $D$ is the true displacement.

measured displacement $D_{i}$ should be divided by cosine value of their included angle to become the true displacement $D$. In case $2, \vec{M}$ is parallel to $\vec{L}$, but not parallel to $\vec{D}$. In case $3, \vec{L}$ is parallel to $\vec{D}$, but not parallel to $\vec{M}$. The true displacement can be obtained after appropriate compensation according to the misalignment configuration.

The optical configuration near the optical fiber's end is illustrated in Fig. 9. The optical fiber emits light in a cone shape. The cone angle, the critical angle $\theta_{C}$, is dependent on the refraction indexes of the core and cladding materials of the optical fiber. In this paper, we use SMA-28 single-mode optical fiber, and its critical angle $\theta_{C}$ is $7.5^{\circ}$. In practice, we cannot align the optical fiber perpendicularly straight to the mirror. An included angle $\delta$ is assumed in Fig. 9. If and only if $\delta$ is less than or equal to $\theta_{C}$, the reflected light can be coupled back to the fiber, and thus, the interference will happen. It is very easy to ensure the misalignment angle $\delta$ to be less than or equal to $7.5^{\circ}$, since a clear interference signal is received by the detector. Comparing Fig. 8 to Fig. 9, we can see Fig. 9 matches with case 2 in Fig. 8. The measurement error only comes from the included angle $\beta$ between $\vec{M}$ and $\vec{D}$.

To minimize the measurement error, we glue the mirror directly to the end surface of the piezoelectric actuator. The end surface is well polished, and the manufacturer guarantees that $\beta$ is less than $0.1^{\circ}$. Therefore, the relative error is less than $1-\cos \left(0.1^{\circ}\right)$, which is $1.5 \mathrm{ppm}$.

Actually, major measurement error is caused by the thermal drift in laser diode rather than the misalignment. Undesired thermal drift may cause $A(x)$ and $D(x)$ to vary with 


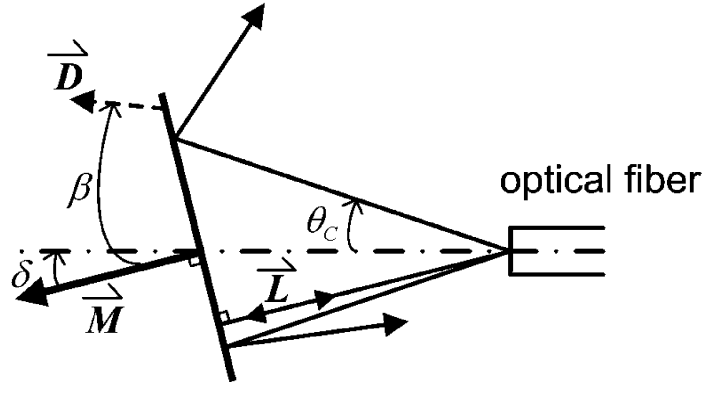

Fig. 9. Optical configuration near the optical fiber's end. $\vec{L}$ is the propagation vector of light; $\vec{M}$ is the normal vector of the measured surface; $\vec{D}$ is the displacement vector of the measured surface; and $\theta_{C}$ is the critical angle of the optical fiber.

TABLE I

SPECIFICATIONS OF THE PROPOSED FFPI

\begin{tabular}{cccc}
\hline \hline & Range & Resolution & Speed \\
\hline Coarse Mode & $\mathbf{1 m m}$ & $164 \mathrm{~nm}$ & $1 \mathrm{~mm} / \mathrm{s}$ \\
Fine Mode & $164 \mathrm{~nm}$ & $\mathbf{\pm 5 n m}$ & $20 \mu \mathrm{m} / \mathrm{s}$ \\
Scheduling Mode & $\mathbf{1 m m}$ & $\mathbf{\pm 5 n m}$ & $20 \mu \mathrm{m} / \mathrm{s}$ \\
\hline
\end{tabular}

time, and thus, influence the measurement accuracy. In order to deal with this problem, a high-class laser stabilizer (WLD3343, Wavelength Electronics, Inc.) is equipped in our system. With a large heatsink to suppress the temperature rise of the laser element and automatic laser intensity control provided by the stabilizer, the variance of the wavelength is $1310 \pm 4 \mathrm{~nm}$ over $25 \pm 5{ }^{\circ} \mathrm{C}$ temperature fluctuation. Under this condition, the relative measurement error is limited within $\pm 0.3 \%$.

\section{CONCLUSION}

In this paper, we designed a novel modulation/demodulation scheme to extend the measurement range beyond the limit of the wavelength of interferometer successfully. This technique combines the coarse (digital signal) and fine mode (analogy signal) in FFPI. Referring to Table I, the constructed FFPI can operate in coarse and fine modes, which have very different characteristics. The coarse mode's resolution is not very high but it has faster response and longer measurement range than the fine mode. Therefore, we design a fast PI controller for coarse mode and an accurate adaptive sliding controller for fine mode. Furthermore, a scheduler is designed to integrate these two modes so that the overall system has a long positioning range (full stroke of the actuator, $70 \mu \mathrm{m}$ ) and a high resolution $( \pm 5 \mathrm{~nm})$.

Theoretically, the measurement range should be limited by the coherence of the laser source. Using the widely used semiconductor laser, the measurement range of our system is examined to reach $1 \mathrm{~mm}$. Beyond $1 \mathrm{~mm}$ of the air gap, the signal-to-noise ratio is too low so that the accuracy cannot be guaranteed. On the other hand, better resolution can be expected if even shorter wavelength is adopted.

\section{ACKNOWLEDGMENT}

The authors would like to thank Taiwan Precision Instrument (TPI) Company, Ltd. for valuable technical supports.

\section{REFERENCES}

[1] T. Wang, S. Zheng, and Z. Yang, "A high precision displacement sensor using a low-finesse fiber-optic Fabry-Perot interferometer," Sens. Actuators A., vol. 69, pp. 134-138, 1998.

[2] G. B. Hocker, "Fiber-optic sensing of pressure and temperature," Appl. Opt., vol. 18, no. 9, pp. 1445-1448, 1976.

[3] K. A. Murphy, M. F. Gunther, A. M. Vengsarkar, and R. O. Claus, "Quadrature phase-shifted, extrinsic Fabry-Perot optical fiber sen-sors," Opt. Lett., vol. 16 , no. 4, pp. 273-275, 1991.

[4] A. Dandridge, A. B. Treven, G. H. Sigel Jr., E. J. West, and T. G. Giallorenzi, "Optical fiber magnetic field sensors," Electron. Lett., vol. 16, no. 11 , pp. 408-409, 1980.

[5] A. Vali and R. W. Shorthill, "Fiber ring interferometer," Appl. Opt., vol. 15 , no. 5 , pp. $1445-1448,1979$.

[6] T. Yoshino, K. Kurosawa, K. Itoh, and T. Ose, "Fiber-optic fabry-perot interferometer and its sensor applications," IEEE Trans. Microw. Theory Tech., vol. 30, no. 10, pp. 1612-1621, Oct. 1982.

[7] U. Schnell, R. Dandliker, and S. Gray, "Dispersive white-light interferometry for absolute distance measurement with dielectric multi-layer systems on the target," Opt. Lett., vol. 21, no. 7, pp. 528-530, 1996.

[8] Y. Y. Cheng and J. C. Wyant, "Multiple-wavelength phase-shifting interferometry," Appl. Opt., vol. 24, no. 6, pp. 804-807, 1985.

[9] P. J. Groot, "Extending the unambiguous range of two-color interferometers," Appl.Opt., vol. 33, no. 25, pp. 5948-5953, 1994.

[10] P.-Y. Chien, Y.-S. Chang, and M.-W. Chang, "Electrically nulled interferometric sensor based on triangular phase modulation," Opt. Commun. vol. 135, pp. 198-202, 1997.

[11] S. A. Meller, "Extrinsic fabry-perot interferometer system using wavelength modulated source," M.A. thesis, Dept. Elect. Eng., Virginia Polytechnic Inst. State Univ., Blacksburg, VA, 1996.

[12] S. Hung and L. Fu, "Design and experiment of range-extended fiber FabryPerot interferometer utilizing the second harmonic displacement modulation," in Proc. IEEE/ASME Int.Conf. Advanced Intelligent Mechatronics, 2005, pp. 88-92.

[13] D. W. Pessen, Industrial Automation. Hoboken, NJ: Wiley, 1989, p. 327

[14] L. V. De Leemput, P. Ronden, B. Timmerman, and H. Van Kempen, "Calibration and characterization of piezoelectric elements as used in scanning tunneling microscopy,” Rev. Sci. Instrum., vol. 62, no. 4, pp. 989-992, Apr. 1991.

[15] S. Moriyama, F. Uchida, and E. Seya, "Development of a precision diamond tuming machine for fabrication of asymmetric aspheric mirrors," Opt. Eng., vol. 27, no. 11, pp. 1008-1012, Nov. 1988.

[16] J. Stroscio and W. Kaiser, Scanning Tunneling Microscopy. New York: Academic, 1993.

[17] W. B. Cook, H. E. Snell, and P. B. Hays, "Multiplex Fabry-Perot interferometer:I. theory," Appl. Opt., vol. 34, no. 24, pp. 5263-5267, 1995.

[18] J. L. Santos, A. P. Leite, and D. A. Jackson, "Optical fiber sensing with a low-finesse Fabry-Perot cavity," Appl. Opt., vol. 31, no. 34, pp. 73617366, 1992.

[19] G. Keiser, Optical Fiber Communications., 2nd ed. New York: McGrawHill, 1991, p. 114.

[20] Manual of Model SR830 DSP Lock-in Amplifier, SRS Inc., Sunnyvale, CA, 2002, pp. 3-1-3-2.

[21] P. A. Ioannou and J. Sun, Robust Adaptive Control. Englewood Cliffs, NJ: Prentice-Hall, 1998

[22] M. Chen, H. Huang, S. Hung, and L. Fu, "Design and implementation of a new 3-DOF electromagnetic micropositioner utilizing flexure mechanism," in Proc. IEEE Am. Control Conf., 2006, pp. 1098-1103.

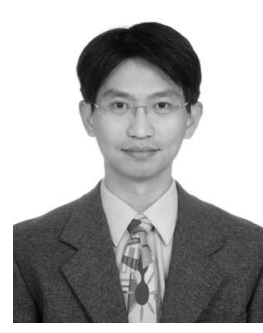

Shao-Kang Hung ( $\mathrm{S}^{\prime} 03$ ) was born in Keelung, Taiwan, R.O.C., in 1976. He received the B.S. and M.S. degrees from the Department of Mechanical Engineering, National Taiwan University, Taipei, Taiwan, in 1998 and 2000, respectively. He is currently working toward the Ph.D. degree in the Department of Electrical Engineering, National Taiwan University.

Since 2000, he has been with the Nanoscience Laboratory, Institute of Physics, Academia Sinica, Taiwan, where he has been involved in the instrumentation of scanning probe microscopy. His current 
research interests include nanoscale instrumentation, optomechatronic systems, precision motion control, and autonomous machine design.

Mr. Hung received the Best Project Award of the National Students' Hands-on Competition in 2003 and the Exceptional Award of the students' paper contest of the Chinese Automatic Control Society in 2005.

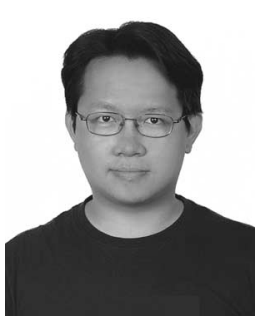

En-Te Hwu received the B.S. and M.S. degrees from the Department of Mechanical Engineering, National Sun Yat-sen University, Kaohsiung, Taiwan, R.O.C., in 1999 and 2001, respectively. He is currently working toward the Ph.D. degree in the Department of Mechanical Engineering, National Taiwan University, Taipei, Taiwan, R.O.C.

Since 2001, he has been with the Nanoscience Laboratory, Institute of Physics, Academia Sinica, Taiwan, where he has been involved in the instrumentation of scanning probe microscopy.

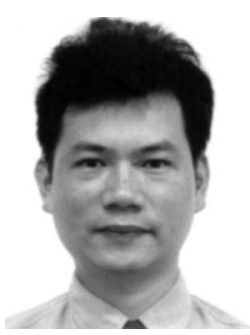

Mei-Yung Chen was born in Maju, Taiwan, R.O.C., in 1966. He received the B.S. degree from the Department of Mechanical Engineering, TamKang University, Taipei, Taiwan, in 1992, the M.S. degree from the Department of Mechanical Engineering, Chung Yuan Christian University, Chung-Li, Taiwan, in 1994, and the Ph.D. degree from the Department of Electrical Engineering, National Taiwan University, Taipei, Taiwan, in 2003 .

$\mathrm{He}$ is currently an Assistant Professor in the Department of Industrial Education, National Taiwan Normal University, Taipei, Taiwan. Li His current research interests include magnetic levitation technology, positioning and tracking, mechatronics, and control theory and applications.

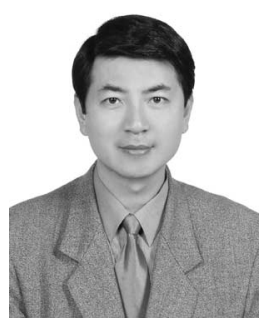

Li-Chen Fu (F'04) was born in Taipei, Taiwan, R.O.C., in 1959. He received the B.S. degree from the Department of Electrical Engineering, National Taiwan University, Taipei, Taiwan, in 1981, and the M.S. and Ph.D. degrees from the Department of Electrical Engineering and Computer Science, University of California, Berkeley, in 1985 and 1987, respectively.

Since 1987 , he has been a member of the faculty and is currently a Professor in both the Department of Electrical Engineering and Department of Computer Science and Information Engineering of National Taiwan University. His current research interests include adaptive control, nonlinear control, precision measurement, visual tracking, control of robots, and FMS scheduling. He has been the Editor of the Journal of Control and Systems Technology and an Associate Editor of Automatica. Since 1999, he has been the Editor-in-Chief of the Asian Journal of Control.

Dr. Fu was a member of the IEEE Robotics and Automation Society from 1996 and 1998 and in 2000, and served as the Program Chair of the 2003 IEEE International Conference on Robotics and Automation and the 2004 IEEE Conference on Control Applications. He received the Excellent Research Award from 1990 to 1993 and Outstanding Research Awards in 1995, 1998, and 2000 from the National Science Council, R.O.C.; the Outstanding Youth Medal in 1991, the Outstanding Engineering Professor Award in 1995, the Best Teaching Award in 1994 from the Ministry of Education; the Ten Outstanding Young Persons Award in the 1999 of the R.O.C.; the Outstanding Control Engineering Award from the Chinese Automatic Control Society in 2000; and the Lee Kuo-Ding Medal from the Chinese Institute of Information and Computing Machinery in 2000. 